\section{Cahiers de Narratologie}

Analyse et théorie narratives

$16 \mid 2009$

Images et récits

\title{
Images documentaires dans le récit décolonisateur. Le cinéma politique latino- américain des années 60 et 70
}

Susana Sel

\section{OpenEdition}

Journals

Édition électronique

URL : http://journals.openedition.org/narratologie/1060

DOI : 10.4000/narratologie. 1060

ISSN : 1765-307X

Éditeur

LIRCES

Référence électronique

Susana Sel, «Images documentaires dans le récit décolonisateur. Le cinéma politique latinoaméricain des années 60 et 70 », Cahiers de Narratologie [En ligne], 16| 2009, mis en ligne le 25 mai 2009, consulté le 30 avril 2019. URL : http://journals.openedition.org/narratologie/1060 ; DOI: 10.4000/narratologie.1060

Ce document a été généré automatiquement le 30 avril 2019

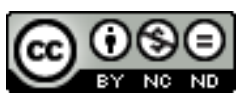

Cahiers de Narratologie - Analyse et théorie narratives est mis à disposition selon les termes de la licence Creative Commons Attribution - Pas d'Utilisation Commerciale - Pas de Modification 4.0 International. 


\title{
Images documentaires dans le récit décolonisateur. Le cinéma politique latino-américain des années 60 et 70
}

\author{
Susana Sel
}

Entre fiction et réalité, la vérité du cinéma

1. Dans la fiction, le récit filmique se définit comme une succession d'images objectives et subjectives dont la combinaison complexe débouche sur l'élaboration de l'identité du personnage. Quand cette distinction entre images objectives et images subjectives est perturbée, comme dans Citizen Kane (Orson Welles, 1941), et qu'elle s'évanouit dans ce que virent les témoins, l'identité du personnage devient problématique. Le récit, selon les termes de Deleuze, ne renvoie plus désormais à un idéal de vérité, mais il devient un pseudo-récit, ou plutôt un simulacre de récit ${ }^{1}$. Eisenstein s'intéresse davantage à la lecture de la réalité ${ }^{2}$. Il conçoit le cinéma comme un instrument (parmi d'autres) de cette lecture : il n'est pas tenu de reproduire «la réalité » tout en se gardant d'agir sur elle, mais au contraire, il doit refléter cette réalité tout en émettant à son égard un jugement idéologique, tout en présentant un discours idéologique. De sorte que pour Eisenstein, le film est moins considéré comme une représentation que comme un discours articulé, soutenu par une référence figurative à la réalité. Sa réflexion sur le montage revient précisément à définir cette "articulation" selon différents axes: le fragment et le conflit; l'extension du concept de montage, l'influence sur le spectateur. Le récit est l'énoncé dans sa matérialité, c'est le texte narratif en charge de raconter l'histoire ${ }^{3}$. Il se présente comme un discours du fait qu'il implique à la fois l'énonciateur et le lecteurspectateur. Et, afin que ce spectateur soit à même de comprendre à la fois l'ordre du discours et l'ordre de l'histoire, il doit établir une cohérence interne en fonction d'une grammaire qui s'impose comme telle au spectateur. Pour Sorlin, le récit implique le spectateur si bien qu'il doit s'immerger dans la projection, accepter passivement ses règles ou les refuser et abandonner la salle de cinéma. L' " histoire » dramatisée condense les situations et les relations et les porte à un degré extrême. Elle crée un univers superficiel et isolé, au sein duquel le spectateur est immergé et qui le prive de toute initiative ${ }^{4}$. De sorte que le combat pour une narration distanciée constitue une donnée 
essentielle des luttes militantes dans le domaine culturel. Birri ${ }^{5}$ définit cette articulation au sein du documentaire social argentin des années soixante:

Le sous-développement est une donnée incontestable de l'Amérique latine, y compris de l'Argentine. C'est un fait économique et statistique. L'expression " sous-développement » n'est pas une invention de la Gauche, des organisations « officielles » internationales (ONU) et latino-américaines (OEA, CEPAL, ALALC) l'utilisent couramment dans les textes de leurs rapports et de leurs plans. Ils ne peuvent pas ne pas l'utiliser. Les causes qu'ils servent sont bien connues : le colonialisme qui s'exerce depuis l'extérieur de l'Amérique Latine et à l'intérieur de ses frontières. Le cinéma de nos pays reprend à son compte les caractéristiques générales de cette superstructure, de cette société, il en assume tous les mensonges. Il donne une image fausse de notre société, de notre peuple, il escamote le peuple : il ne donne pas une image du peuple. En donner une sera un premier pas positif : c'est la fonction du documentaire. Quelle image du peuple le cinéma documentaire offre$\mathrm{t}$-il ? Il ne peut faire autrement que donner une image fidèle à la réalité. (Telle est la fonction révolutionnaire du documentaire social latino-américain). En témoignant de cette réalité - de cette sous-réalité, de ce malheur - il les conteste. Il les renie. Il les dénonce. Il les juge, les critique, les démonte. Car il montre les choses telles qu'elles sont, d'une manière irréfutable, et non pas comme nous voudrions qu'elles soient. (Ou comme - de bonne ou de mauvaise fois - on voudrait nous faire croire qu'elles sont). Le documentaire trouve un contrepoint à cette contestation dans l'affirmation des valeurs positives de notre société: les valeurs du peuple. Sa force, son travail, ses joies, ses luttes, ses rêves. Conséquences - et raisons d'être - du documentaire social : la connaissance, la conscience, la prise de conscience de la réalité. Problématisation. Changement : d'une vie qui n'en est pas une à la vraie vie. Conclusion : disposer une caméra face à la réalité et la documenter, documenter le sous-développement. Le cinéma qui se rend complice du sous-développement est un sous-cinéma ${ }^{6}$.

2 Moyennant des formes diverses, toutes opposées à la fiction, le documentaire va aborder la réalité en empruntant de nouveaux chemins, fidèle à un idéal de vérité, bien que dépendant toujours de la fiction cinématographique (objectif-subjectif).

Ces questions prennent un contour différent lorsque l'on applique au réel un idéal ou un modèle de vérité, la caméra saisissant alors un réel préexistant alors que par ailleurs, les conditions du récit n'ont pas changé : l'objectif et le subjectif subissent un déplacement et non une transformation ; les identités sont définies selon un autre mode ; le récit parvient à acquérir une vraisemblance réelle au lieu d'une vraisemblance fictive. Ce nouveau type de récit n'en reste pas moins une fiction. La césure ne se situe plus désormais entre fiction et réalité, mais s'inscrit au sein même d'un nouveau mode de récit qui les affecte l'une et l'autre. Le concept de « cinéma du vécu » élaboré par Perrault critique la fiction sous toutes ses formes. Perrault lui reproche de produire un modèle de vérité pré-établi qui exprime nécessairement les idées dominantes ou le point de vue du colonisateur, y compris lorsque c'est le réalisateur qui forge ce modèle ${ }^{7}$. Lorsque, au Québec, Perrault s'adresse à ses personnages réels, ce n'est pas seulement pour éliminer la fiction, mais bien pour la libérer du modèle de vérité qui pénètre en elle, et découvrir la «fonction de fabulation » qui s'oppose à ce modèle. Ce qui s'oppose à la fiction, ce n'est pas le réel, ce n'est pas la vérité, qui demeure celle des maîtres et des colonisateurs, mais c'est la fonction fabulatrice des pauvres, qui octroie au faux la puissance d'une mémoire ou d'une légende. Perrault considère que ce que le film doit saisir, ce n'est pas l'identité d'un personnage, réel ou fictif, à travers ses aspects objectifs ou subjectifs, mais le devenir du 
personnage réel lorsqu'il se met à "fictionnaliser », lorsqu'il se laisse surprendre "en flagrant délit de légende » et qu'il contribue ainsi à l'invention de son peuple. Il n'est pas possible de séparer le personnage d'un avant et d'un après. Il incarne le passage d'un état à l'autre. De son côté, le cinéaste aussi devient un autre lorsqu'il contribue à la transformation des personnages réels et qu'il remplace la fiction par la fabulation. Cinéaste et personnages communiquent en inventant un peuple. C'est la simulation d'un récit, d'une légende et de ses métamorphoses, c'est le discours indirect libre du Québec. C'est dans ces conditions que le cinéma peut s'appeler cinéma-vérité, avec d'autant plus de raisons qu'il aura détruit tout modèle de vérité, pour se faire créateur, producteur de vérité : ce n'est pas le cinéma de la vérité, c'est la vérité du cinéma. Perrault produit son discours indirect libre en même temps que ses personnages produisent celui du Québec. Pour le réalisateur, il s'agit d'appartenir à un peuple dominé et de recouvrer une identité collective perdue, réprimée.

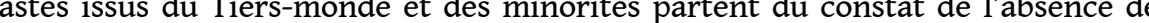
peuple. D'où leur ambition de contribuer à son invention. Alors que le maître et le colonisateur proclament qu' «il n'y a jamais eu de peuple ici! », le peuple absent est un devenir, il s'invente, dans les quartiers pauvres, grâce à de nouvelles formes de luttes auxquelles un art nécessairement politique doit contribuer.

Le nouveau cinéma latino-américain

5 Dans les années soixante, le concept de Tiers-monde réunit les opposants à l'impérialisme. Marquée par l'expansion du processus de décolonisation en Afrique, par le triomphe de la révolution cubaine en 1959, par la naissance du Mouvement des pays nonalignés, lors de la Conférence de Bandung (1955), il gagne désormais trois continents, l'Afrique, l'Amérique Latine et l'Asie. L'expérience née des divers processus de décolonisation se fortifie dans un tiers-mondisme qui transcende le caractère national des mouvements de libération. Les luttes nationales contre l'impérialisme coïncident avec l'exacerbation de la lutte des classes à l'intérieur de chacun des pays concernés.

C'est dans ce contexte que surgit, dans les années soixante, la catégorie de «nouveaux cinémas ", pour désigner les courants de rénovation cinématographique qui se déploient de l'Europe (avec le Nouveau cinéma allemand, la Nouvelle Vague française, le Free Cinema anglais) jusqu'à l'Amérique Latine (avec le Cinéma cubain et le Cine Novo brésilien). Ces courants très divers contestent le cinéma commercial hollywoodien, dont la production se fonde sur des codes qui sont ceux de l'« american way of life » et dont les objectifs sont exclusivement mercantiles. Les nouveaux cinémas latino-américains s'efforcent de renouer avec une réflexion de la société sur elle-même, selon les principes hérités du Néo-réalisme italien, ce courant artistique qui, entre 1945 et 1965, avait imposé, dans une Italie dévastée par la guerre, une nouvelle façon de faire du cinéma. Dans un contexte de misère, de famine, de chômage, les réalisateurs rompent avec les schémas traditionnels, en installant leurs caméras hors des studios, dans les logements humbles et les quartiers populaires, en choisissant leurs acteurs parmi les gens de la rue et en s'identifiant à leurs problèmes.

7 Le Nouveau Cinéma Latino-américain assume l'héritage du cinéma néoréaliste. Le Nouveau Cinéma Latino-américain comme le Néo-réalisme sont deux cinémas de dénonciation apparus dans des contextes semblables, créés par une situation sociale également dégradée, même si le premier, contrairement au second, n'est pas une réponse à la guerre, mais une contestation de la domination des oligarques et des militaires à la solde des Etats-Unis. Le Nouveau Cinéma Latino-américain s'inscrit dans une période qui 
s'étend pratiquement du triomphe de la Révolution cubaine (1959) jusqu'au début des dictatures des années 70 . Ses manifestes écrits et cinématographiques dénoncent les atteintes aux droits de l'homme et rendent l'impérialisme nord-américain responsable des crises qui affectent le sous-continent. L'arrivée à Cuba de réalisateurs italiens au début du nouveau cinéma cubain et les séjours d'étude en Italie effectués par la plupart des cinéastes latino-américains créent une relation de parenté entre ces deux formes de cinéma social, dépourvus de moyens économiques (dans le cas italien, la précarité économique n'est réelle qu'au début ${ }^{8}$ ), et font naître des formes narratives semblables.

Le Nouveau Cinéma Latino-américain a produit en images un discours d'appartenance à une culture spécifique, à une identité spécifique, avec pour objectif un cinéma critique, social, susceptible, dans les années soixante et au début des années soixante-dix, de rencontrer les désirs politiques et culturels de spectateurs soucieux de repenser leur propre réalité. Lorsque la situation politique l'a permis, certains films ont pu intégrer les circuits commerciaux, mais la majorité d'entre eux n'a connu qu'une diffusion clandestine dans les usines, les universités, les communautés paysannes. Les festivals du Nouveau Cinéma Latino-américain sont devenus des lieux de militantisme, où l'on pouvait voir les nouvelles productions et surtout où les cinéastes militants latinoaméricains se retrouvaient autour d'une lutte commune contre l'impérialisme, une lutte qui les distinguait d'autres courants cinématographiques. Le festival fondateur du Nouveau Cinéma Latino-américain se tient à Viña del Mar (Chili) en 1967. Il réunit, pour la première fois, des cinéastes argentins, boliviens, brésiliens, colombiens, cubains, mexicains, péruviens, uruguayens, vénézuéliens et chiliens. Les films projetés, des productions indépendantes dont les objectifs sont culturels, artistiques, didactiques et politiques, ont une existence en marge de la distribution commerciale. Ce premier festival latino-américain, consacré principalement au cinéma documentaire, connaîtra une nouvelle édition en 1969 qui inclura dans sa programmation les longs-métrages de fiction. Entre ces deux éditions, le cinéma du sous-continent latino-américain a évolué. Il a enregistré, en particulier, l'émergence de cinéastes d'envergure mondiale, comme les Brésiliens Glauber Rocha et Nelson Pereira Dos Santos, les Argentins Fernando Solanas et Octavio Getino, les Cubains Santiago Alvarez et Tomás Gutiérrez Alea, les Chiliens Raúl Ruiz et Miguel Littin. La troisième édition du festival prévue en 1973 sera annulée en raison du coup d'état de Pinochet.

9 Au cours des années 70, la situation politique interdit la circulation de ce genre de cinéma. Pire, nombreux sont les cinéastes persécutés en Argentine, au Chili, au Brésil, en Uruguay, en Bolivie, en Colombie. L'exil, la torture, la mort, tel est le sort que leur réservent les dictatures latino-américaines. Les cinéastes argentins Raymundo Gleizer et de Pablo Szir comptent parmi les «disparus » en 1976. Les Chiliens Jorge Müller et Carmen Bueno avaient connu le même sort en 1974. D'autres sont purement et simplement assassinés comme l'emblématique caméraman argentin Leonardo Henrichsen, tué par une patrouille militaire non loin du Palais présidentiel où il était entrain de filmer le soulèvement militaire qui préfigurait le coup d'état de Pinochet ${ }^{9}$.

Le nouveau Cinéma Cubain

10 La production du Nouveau Cinéma Latino-américain, irriguée par les diverses avantgardes européennes, fut elle-même variée. Ainsi la narration subjective mise en œuvre par Santiago Alvarez à Cuba puise aux sources du documentaire soviétique de Dziga Vertov, des théories du montage dues à Serguei Eisenstein, du cinéma de Chris Marker ou de Joris Ivens. Pour Santiago Alvarez, chaque cinéaste possède ses propres idées et une 
manière bien à lui de les transmettre. Et ces idées se nourrissent de la confrontation politique.

11 Santiago Alvarez ne se contente pas de réaliser les premiers documentaires politiques du Nouveau Cinéma Latino-américain, en associant les objectifs déjà mentionnés à l'expérimentation de nouvelles formes de narration. Il est le fondateur et le directeur des

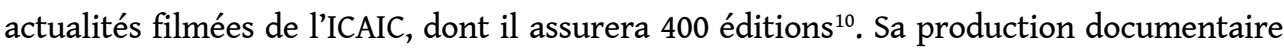
porte une forte empreinte journalistique. Elle est marquée par des événements historiques importants comme la guerre du Vietnam, la révolution chinoise, la figure de Ho-Chi-Min, la tentative d'invasion de Cuba (Playa Girón en 1961). Dans ses films, l'action dramatique s'appuie sur un art consommé du montage et sur l'usage de la bande son. Alvarez explique que ses innovations, sa manière de «couper et coller des bouts de films ", comme il dit, ont surgi en réaction aux actualités filmées telles qu'elles existaient auparavant. A ses yeux, le cinéma, loin de n'être qu'une affaire de style, relève d'une question idéologique et requiert une prise de position déterminée, une attitude militante, d'autant plus nécessaires dans des pays sous domination. « Les armes et le combat sont des mots qui effraient, mais le problème pour les cinéastes est de s'imprégner de la réalité actuelle, de prendre son pouls. C'est ainsi que s'évanouit la peur des mots chargés de contenus péjoratifs à laquelle cède très souvent le créateur ${ }^{11}$. » Ces propos annoncent les définitions du Tiers-cinéma qui, quelques années plus tard, seront proposées par les Argentins Solanas et Getino.

12 L'un des documentaires paradigmatiques de Santiago Alvarez a pour titre Now ${ }^{12}$ (1965). Anticipant une forme qui allait devenir celle du clip vidéo, l'association d'images d'archive (des extraits de films d'actualités et des photographies) et de la version anglaise de la chanson israélienne Hava Nagila, interprétée par Lena Horne, sert un impressionnant plaidoyer antiraciste de six minutes. Le recours à la photographie animée et au collage de documents d'archives montre, dès le début du film, de quelle manière et avec quelle brutalité la police américaine tente de briser la résistance de la population afroaméricaine, tandis que la musique qui accompagne ces images se charge d'une valeur critique. Aux yeux d'Alvarez, ce type de discours s'apparente à une forme de lutte contre la discrimination raciale. Dans le même esprit, il sollicite les figures fondatrices de la nation américaine comme Jefferson, Washington, Lincoln comme pour en appeler aux principes démocratiques, constitutifs de la nation. Il reprend une série d'images de violences et de tortures infligées à la population noire des Etats-Unis pour terminer sur un carton sur lequel s'inscrit le mot NOW qui apparait projeté comme une réponse frontale à cette forme de violence. Pour Santiago Alvarez,

La réalité convulsive qui est la nôtre, celle que vit le Tiers-monde, exige de l'artiste qu'il se fasse violence, qu'il se laisse consciemment emporter par une tension créatrice. Sans idées préconçues, sans préjugés, sans rabaisser l'art ni faire de la pédagogie, l'artiste doit entrer en communication avec le peuple et contribuer à son éveil culturel ${ }^{13}$.

13 Tomás Gutiérrez Alea compte parmi les réalisateurs importants du Nouveau Cinéma Cubain. L'un de ses longs-métrages, Memorias del subdesarrollo (Mémoire du sousdéveloppement, 1968, 85 minutes) défie avec ironie les contradictions de la bourgeoisie. En retraçant le parcours d'un individu pendant la révolution, il parvient à montrer la solitude et la détresse de ceux qui décident de rester en marge de l'Histoire.

Le Tiers-cinéma argentin

14 Dans la continuité de l'action engagée par le Nouveau Cinéma Cubain et dans le contexte des mouvements de libération du Tiers-monde, émerge un "Tiers-cinéma» de 
décolonisation culturelle. Ses œuvres s'inscrivent dans un processus de libération, face à un « Premier-cinéma » identifié au modèle hégémonique hollywoodien dont l'objectif est la conservation du système et à un "Deuxième-cinéma " qui revendique la notion d'auteur depuis une perspective réformiste et qui aspire en réalité à accéder au marché $\mathrm{du}$ « Premier cinéma ».

Le Tiers-cinéma oppose au cinéma industriel un cinéma artisanal ; au cinéma des individus, un cinéma des masses ; au cinéma d'auteur, un cinéma des collectifs ; au cinéma de désinformation néocolonial, un cinéma d'information ; au cinéma d'évasion, un cinéma qui restaure la réalité ; au cinéma passif, un cinéma agressif ; au cinéma institutionnalisé, un cinéma de guérilla, au cinéma spectacle, un cinéma d'action, au cinéma de destruction, un cinéma à la fois de destruction et de construction; au cinéma fait par l'homme du passé et pour l'homme du passé, un cinéma à la mesure de l'homme nouveau.

Ainsi s'expriment Solanas et Getino en $1973^{14}$.

Plusieurs groupes et réalisateurs empruntèrent le chemin tracé par le cinéma politique et militant argentin et firent de l'intervention politique l'objectif de leur activité cinématographique. On distingue principalement deux tendances : l'une représentée par le Cinéma Libération (Fernando Solanas et Otavio Getino) et l'autre dite du Cinéma de la Base (Raymundo Gleyzer).

17 La Hora de los hornos (L'Heure des brasiers, Solanas et Getino, 1965-1968) qui bénéficie d'une sortie internationale en 1968, lors de la quatrième édition du Festival International du Nouveau Cinéma de Pesaro, inaugure ce courant cinématographique. L'Institut du Cinéma qui subventionne pratiquement toute la production argentine refuse le scénario de $L a$ Hora de los hornos tel qu'il lui est présenté en 1966. Une décision qui anticipe le coup d'état qui va se produire quelques mois plus tard entraînant l'interdiction de toute exploitation commerciale du film jusqu'en 1973. Le film compte trois parties: "Néocolonialisme et violence » (d'une durée de 90 minutes) ; "Action pour la libération » (110 minutes) et «Violence et libération » (45 minutes). Il fut réalisé dans la clandestinité sur une période de deux ans au cours de laquelle les réalisateurs parcoururent le pays pour enregistrer de nombreux entretiens. Le tournage était effectué par Solanas lui-même à l'aide d'une caméra en $16 \mathrm{~mm}$ dépourvue de son synchronisé. C'est la société de production de publicité de Fernando Solanas qui assura le financement du projet. Le montage fut effectué sur une moviola manuelle et avec un vieux projecteur en $16 \mathrm{~mm}$.

Le film adopte la structure de l'essai (il a pour sous-titre Notes sur le néocolonialisme et la violence), et se divise en chapitres et en notes. Il repose sur un important travail de montage afin que les idées contenues dans les notes puissent être réunies dans une synthèse finale. Solanas et Getino expliquèrent qu'ils avaient filmé «en utilisant la pellicule comme un carnet et la caméra comme un stylo ». La première des trois parties, " Néocolonialisme et violence », est la plus novatrice du point de vue du langage. Elle est également la mieux connue internationalement. L'Histoire de l'Amérique Latine y est présentée comme l'histoire de la succession des dominations coloniales, de la domination espagnole à la domination britannique, de la domination britannique au «néocolonialisme» des Etats-Unis. En Argentine, cette violence néocoloniale vise les paysans sans terre, la classe des travailleurs opprimés et tous ceux qui contestent le modèle qu'elle impose par la force. Un chapitre particulier est consacré à la bourgeoisie et aux intellectuels argentins, présentés comme des traitres, aliénés et endormis par les moyens de communication de masse. Dans sa conclusion, le film explique que la seule 
solution pour les peuples latino-américains, c'est de faire le choix de vivre et de mourir pour la révolution contre l'impérialisme. Un plan de quatre minutes sur le visage sans vie de Che Guevara, accompagné par la musique des tambours indigènes, clôt avec solennité la première partie de La Hora de los hornos.

Le récit a recours à un certain nombre d'effets qui visent à faire en sorte que le message politique produise une émotion chez le spectateur. Les intertitres ( LL'histoire qu'ils nous ont enseignée est fausse ", "Un peuple sans haine ne peut triompher ", "Aucun ordre social ne se suicide ») renouent avec l'esthétique des cartons du cinéma muet et retrouvent l'impact des messages publicitaires. A partir de la théorie du montage intellectuel d'Eisenstein, les idées pénètrent dans des séquences chargées d'émotions, destinées à produire chez le spectateur la sensation d'un conflit à la fois affectif et intellectuel. C'est ainsi que la bête robuste présentée dans un concours agricole devient métaphore de la grande propriété terrienne ou que des images d'abattoir alternent avec des plans sur des icônes publicitaires ou avec des images de répression policière. S'appropriant aussi bien la distanciation brechtienne que le néoréalisme de De Sica, le film a recours au message subliminal, aux métaphores, à la caricature, aux témoignages, à la musique afin de produire de l'émotion, comme dans une séquence où des images d'une partie de golf illustrent l'Histoire de l'oligarchie. Bien que leurs propos soient différents, toutes les séquences se veulent didactiques afin de provoquer une prise de conscience de la réalité de l'oppression et de déboucher sur un appel à l'action.

En ce sens, La Hora de los hornos s'adresse au spectateur non pas comme à un sujet qu'il convient de divertir, mais comme à un militant potentiel avec lequel il faut débattre. Ainsi la deuxième partie, "Action pour la libération », consacrée aux mouvements de résistance argentins, et la troisième partie "Violence et libération", dédiée à l'argumentation idéologique, comportent-elles des écrans noirs qui permettent d'interrompre la projection afin que s'engage le débat et pour que les groupes militants puissent adapter le matériel destiné à la projection à leur expérience. De sorte qu'il existe plusieurs versions du film selon le principe de l'œuvre ouverte, susceptible d'être modifiée en fonction des circonstances politiques. Lorsque le film ne pouvait être projeté que dans la clandestinité, dans des locaux syndicaux, dans des appartements ou à la campagne, il impliquait le spectateur. Faire le choix de le voir, c'était assumer un risque. D'une certaine manière, c'était s'engager dans le "projet de libération». Dans un entretien avec Godard, Solanas affirme : « Un film sur la libération, c'est-à-dire sur une étape inachevée de notre histoire, ne peut être qu'un film inachevé, un film ouvert sur le présent et sur le futur de la libération. Il revient aux protagonistes de le poursuivre et de le compléter ${ }^{15}$.»

Le long-métrage de fiction dans le Cine Novo brésilien

21 Bien qu'il ait produit des documentaires, le Cine Novo brésilien s'efforça surtout de fictionnaliser les problèmes du Brésil et en particulier la question des antagonismes entre riches et pauvres. Nelson Pereira Dos Santos ouvrit le cinéma brésilien à de nouvelles formes narratives inspirées du documentaire britannique et du néoréalisme italien. Vidas secas (Sécheresse, 135 minutes, 1963), réalisé dans le cadre d'une coopérative de cinéma, est son film emblématique. S'inspirant d'un livre de l'un des plus grands écrivains brésiliens, Graciliano Ramos, Nelson Pereira Dos Santos réalise un film qui associe la famine à la tragédie de la pauvreté, la soumission à l'ignorance, la cruauté à l'austérité des paysages. Sans rechercher d'effets, la caméra témoigne de conditions humaines infiniment dures. Elle capte les images d'hommes et de femmes secs qui respirent, 
mangent, parlent, travaillent et vivent à peine, traversant le Nordeste comme de misérables parias dans un voyage sans début ni fin.

Tourné dans le Sertao, une région très peu peuplée de l'état de Alagoas, le film s'appuie sur une photographie d'inspiration réaliste qui parvient à rendre compte de la réalité du milieu et la précarité des individus. Il décrit l'existence d'une famille qui s'est installée dans le Sertao durant la saison des pluies et qui reprendra son chemin dès le retour de la saison sèche. La narration repose sur des mouvements de caméra très lents et des dialogues minimalistes afin de souligner la monotonie et la précarité de cette existence. La largeur des cadrages, la fixité de la caméra, la longueur des plans, le recours aux effets de caméra subjective, la lenteur de l'action donnent au film l'apparence d'un documentaire. Les rares actions s'inscrivent dans des plans d'une longueur inhabituelle, ce qui semble accroître la durée du film. Dès le début, s'impose la lenteur du temps du récit. L'ellipse est l'un des moyens narratifs que se donne le film pour faire éprouver le temps, comme, par exemple, lorsque le passage d'oiseaux signale le temps qui passe entre la scène où l'une des protagonistes pleure tout en préparant le repas et celle où elle puise de l'eau dans une rivière presque asséchée.

Le récit adopte une temporalité cyclique. Il débute sur l'évocation de l'arrivée de la famille au Sertao et s'achève sur son retour au Sertao. Si les premiers plans décrivent des êtres qui envisagent l'avenir, le dernier plan, filmé en caméra fixe, les montrent tandis qu'ils s'éloignent vers une destiné qui est un éternellement recommencement. Le Cine Novo, désireux de réaliser un cinéma tourné vers la réalité et émancipé du modèle hollywoodien imposé par les grandes sociétés de production, invente des chroniques néoréalistes et se met en quête d'un style national, comme il en existe un en littérature, en peinture, en architecture et en musique. « Nous voulions décoloniser culturellement le Brésil avec un langage qui n'appartiendrait qu'à nous ${ }^{16}$. "

Dans cette même perspective - celle du Cine Novo-, Glauber Rocha critique l'acculturation et souligne les valeurs de ce nouveau cinéma qui rompt avec les canons et les codes narratifs conventionnels sans renoncer à l'ironie ni à l'humour. Son anticonformisme et son sens de l'engagement lui permettent de composer une filmographie qui, sans renoncer à son style novateur ni à son caractère nationaliste, se reconnaît de multiples héritages. Il conjugue les théories du montage d'Eisenstein, le cinéma de Visconti, le spaghetti-western de Sergio Leone, la poésie du Nordeste brésilien et le documentaire social dans le contexte d'une région culturellement imprégnée de l'opéra de Villa-Lobos et des mélodies populaires ${ }^{17}$. Sa dénonciation des conditions imposées par le colonialisme tout comme son combat contre les structures mentales et économiques du sous-développement sous-tendent son investissement dans le Cine Novo qu'il entend impulser aux côtés notamment de Nelson Pereira dos Santos, Ruy Guerra et Carlos Diegues.

Le Cine Novo ne peut connaître de développement réel tant qu'il demeure en marge du processus économique et culturel du continent latino-américain. Par ailleurs, le Cine Novo n'est pas un phénomène circonscrit au Brésil. C'est un mouvement lié aux peuples colonisés : dès lors qu'un cinéaste est prêt à filmer la réalité et à affronter les normes hypocrites et policières de la censure, le Cine Novo peut éclore. Dès lors qu'un cinéaste est disposé à s'opposer aux dictats commerciaux, à l'exploitation, à la pornographie, à la dictature de la technique, le Cine Novo peut éclore. Dès lors qu'un cinéaste, quels que soient son âge ou ses origines, s'apprête à mettre son œuvre et son métier au service des causes essentielles de son temps, le Cine Novo peut éclore. Telle est la définition du Cine 
Novo, une définition qui l'écarte de l'industrie, car le cinéma industriel n'a d'autres alliés que le mensonge et l'exploitation. L'intégration économique et industrielle du Cine Novo dépend de la liberté que saura conquérir l'Amérique Latine. Le Cine Novo lutte pour cette liberté. C'est une question morale dont ses films seront le reflet, dans le temps qu'ils se donneront pour filmer un homme ou une maison, dans les détails de l'observation, dans la philosophie qui sera la leur : ce n'est pas un film mais un ensemble de films qui donnera enfin au public la conscience de sa propre existence. Nous n'avons pas davantage de point de contact avec le cinéma mondial. Le Cine Novo est un projet qui se réalise dans le contexte de la politique de la faim et qui en endure toutes les faiblesses ${ }^{18}$.

C'est dans le cadre d'une défense et illustration de cette "esthétique de la faim" que Glauber Rocha réalise ses deux films les plus importants, Deus e o Diabo na terra do sol (Dieu noir et Diable blond, 1964) et Antonio das Mortes (1969), dans lesquels il expose les conflits liés à la terre. Le récit repose sur la stratégie du contrepoint: d'un côté, le peuple apparait comme une masse qui résiste, mais aussi qui chante et danse jusqu'à l'épuisement afin d'oublier la faim; de l'autre le film décrit un groupe hétérogène de militaires, de représentants de l'Eglise, de policiers unis dans la défense des intérêts des grands propriétaires. Entre les deux, les conflits intérieurs d'un mercenaire, Antonio das Mortes.

Le groupe bolivien Ukamau

26 Le cinéma de Jorge Sanjinés, qui s'inscrit dans cette même recherche d'une identité propre et d'une forme de narration associée à cette identité, se veut en phase avec une nouvelle société imprégnée des savoirs ancestraux et capable de s'adapter aux rythmes internes de la spiritualité nationale. La culture andine et son potentiel révolutionnaire exigeaient de rompre avec l'acculturation et de se tourner vers des codes de communications spécifiques. Jorge Sanjinés est, avec Oscar Soria, à l'origine du groupe UKAMAU qui fonde la première Ecole de Cinéma Bolivienne (Escuela Fílmica Boliviana, 1960) et le Ciné Club Bolivien ${ }^{19}$.

Ukamau (1966), un long-métrage en noir et blanc, fait le récit d'une vengeance ou.comment une histoire d'amour se termine en tragédie. En l'absence de son mari parti pêcher sur le lac Titicaca, Sabina est agressée par Rosendo Ramos, le Métis qui achète les produits des paysans de l'île. La poursuite silencieuse du meurtrier, interrompue par les pleurs de celui qui crie vengeance, s'achève dans l'altiplano sur un corps à corps sanglant. Pour Sanjinés, il est clair que les films ne peuvent pas faire la révolution. En revanche, ils peuvent contribuer à des changements révolutionnaires. Ce fut le cas dans une affaire de stérilisation de populations indigènes qui vit les coupables nord-américains expulsés de Bolivie en 1971 après que le Congrès et l'Université se furent assurés que les faits dénoncés par le film Sangre de Cóndor (Yawar Mallku, 1969) étaient avérés ${ }^{20}$. Sangre de cóndor dont les interprètes sont les membres du collectif Ukamau s'achève sur une image qui montre un groupe de personnes brandissant des armes, comme un symbole de la lutte contre l'oppression et la guerre de classes dont il se fait l'écho. Une image en conclusion d'un film qui n'aura cessé d'opposer la richesse des puissants, installés dans des résidences luxueuses, à la misère du peuple qui vit dans des masures, qui doit voler pour acheter des médicaments, et qui se voit finalement condamné à mort en raison de l'incompréhension des médecins. La praxis démontrait alors qu'un cinéma révolutionnaire pouvait être une arme.

Cependant, même si la situation en Bolivie permettait qu'une œuvre engagée mobilise l'opinion publique et réunisse une large audience, le degré d'invraisemblance du cinéma 
de fiction continuait à rendre impossible la réalisation d'un cinéma populaire susceptible d'aborder les faits réels.

31 Avec El coraje del pueblo (Le courage du peuple, 1971) le groupe tente de donner corps à un cinéma documentaire révolutionnaire. Basé sur des faits réels et documentés et avec la participation comme acteurs d'un nombre important de victimes, le film reconstruit, dramatiquement et en détail, le terrible massacre de la nuit de San Juan qui eut lieu en 1967 et dont l'objectif était d'empêcher mineurs, ouvriers d'usines et étudiants de se réunir pour appuyer la guérilla de Che Guevara et de ses compagnons qui combattaient alors dans la forêt de Nancahuazú. La construction narrative adopte des formes spécifiques à la culture du pays. El coraje del pueblo fut considéré comme le meilleur film présenté lors de la septième Mostra Internazionale de Pesaro (1971). Il obtint les prix OCIC lors du Festival International de Cinéma de Berlin en 1972 et, en 1979, la « Kantuta » d'argent qui récompense le meilleur film bolivien.

Toujours en quête de structures narratives en adéquation avec les perceptions spatiotemporelles du spectateur andin, El Enemigo principal (Le principal ennemi, 1973) reconstitue l'histoire d'un paysan de la communauté Tinkuy, assassiné par un propriétaire terrien pour avoir interpellé la justice suite à un vol de bétail. Ce film auquel participe des ouvriers, des paysans et des étudiants latino-américains, rompt avec les normes dramaturgiques typiques du cinéma occidental. Le surgissement d'un groupe de guérilleros qui exécute le propriétaire et ses hommes de main conduit le conflit vers une sorte de paroxysme. La répression se déchaîne. Les paysans deviennent les victimes de cette lutte entre deux forces opposées qui prétendent les défendre. Le récit élude l'intrigue et s'émancipe des conventions du suspense en introduisant la présence d'un narrateur dont les propos anticipent les événements. De sorte que le spectateur peut les suivre avec une attitude distanciée et raisonnée. Enfin, le film élimine tout protagoniste individuel au profit d'un protagoniste collectif, la communauté.

La Batalla de Chile (La Bataille du Chili)

Réalisée par Patricio Guzmán, la trilogie de 272 minutes intitulée La Batalla de Chile (La Bataille du Chili, 1972-1979) apparaît comme un remarquable document historique tourné au cœur des événements qui précèdent le renversement du gouvernement démocratique du Président Salvador Allende ${ }^{21}$. La caméra de Jorge Müller (également directeur de la photographie) enregistre dans les usines, au sein des syndicats, dans la rue, les témoignages de travailleurs enthousiasmés par le projet politique du gouvernement de l'Unité Populaire ainsi que les manifestations des opposants intransigeants et des militaires putschistes. De mars à septembre 1973, Gauche et Droite s'affrontent dans la rue, dans les usines, devant les tribunaux et au Parlement. Allende tente, sans y parvenir, de passer un accord avec le centre, la Démocratie Chrétienne. A Valparaiso, les militaires fomentent le coup d'état. Un large secteur de la classe moyenne appelle à un boycott économique et politique, dans un climat de guerre civile. Une semaine avant le coup d'état, un million de sympathisants rendent un dernier hommage à Allende. La batalla de Chile est le seul documentaire consacré à la dernière année du gouvernement du président Allende, filmé par une équipe chilienne qui se fait le témoin d'événements exceptionnels dont aucune autre source d'information n'a pu rendre compte. La situation révolutionnaire est filmée jour après jour, au fur et à mesure qu'elle évolue, de même que sont enregistrées les méthodes fascistes auxquelles l'impérialisme et la bourgeoisie ont recours afin de renverser le gouvernement populaire. 

bourgeoisie » : les grèves menées par la Droite et les pressions économiques exercées par les Etats-Unis paralysent les profondes réformes lancées par le président Salvador Allende; malgré cela, l'Unité Populaire recueille $43,3 \%$ des voix lors des élections législatives de 1973 ; l'opposition parlementaire met en œuvre sa stratégie de coup d'état. La deuxième partie s'intitule «Le coup d'état » (1976, 90 minutes) : Gauche et Droite se font face tandis que les Etats-Unis soutiennent financièrement la grève des camionneurs et attisent le chaos social. Allende tente de parvenir à des accords politiques, mais les contradictions internes à la Gauche aggravent la crise; A Valparaiso, les militaires, appuyés par les classes moyennes, préparent le coup d'état. Quelques jours avant son déclenchement, des milliers de sympathisants se réunissent autour du Président Allende ; le 11 septembre Pinochet fait bombarder le palais présidentiel. La troisième partie, « Le pouvoir populaire » (1979, 82 minutes), revient sur des événements importants survenus entre 1972 et 1973 et évoqués dans les deux premières parties : elle montre comment de larges secteurs de la population engagent des actions de soutien au Président Salvador Allende, afin de trouver des réponses au chaos politique et à la crise économique.

Le réalisateur n'envisage pas la question de l'objectivité du documentaire. Pour Patricio Guzmán, ce concept est journalistique et non artistique. A ses yeux, le documentariste est un témoin qui s'implique à travers un regard et un point de vue qui ne peuvent être ignorés. Dans le cadre de cette subjectivité, la vraisemblance du récit est assumée par un discours à la première personne qui s'adresse directement au public dans le but de sauver de l'oubli une mémoire collective.

Aussi bien dans ses séquences d'enthousiasme populaire que dans le tableau qu'il brosse de la réalité douloureuse et convulsive de l'époque, le document en noir et blanc récupère et reconstruit la mémoire collective grâce aux images en mouvement du passé. Il oppose les discours de la Gauche et de la Droite, l'entretien avec l'ambassadeur des Etats-Unis au Chili faisant contrepoint aux idées progressistes de la révolution menée par Allende. Du fait de son parti pris chronologique, le documentaire de Patricio Guzmán remplit une fonction pédagogique pour les nouvelles générations chiliennes en éveillant en elles une conscience critique. L'intervention d'un narrateur qui n'apparaît pas sur les images prises par l'équipe de tournage, ni comme interlocuteur au cours des entretiens permet de compléter certains points particuliers du récit historique.

Après le coup d'état de septembre 1973, Patricio Guzmán est menacé de mort. Deux semaines durant, il est retenu dans l'enceinte du Stade National, privé de toute communication avec l'extérieur,. Il abandonne finalement le Chili, se réfugie à Cuba où il assure le montage du documentaire, avant de s'exiler en Espagne et en France. Son chef opérateur, Jorge Müller, fait partie des « disparus » en 1973.

Repenser la décolonisation par les images documentaires

38 Ce travail ne prétend pas épuiser la réflexion sur la fonction du documentaire dans le Nouveau Cinéma Latino-américain pas plus qu'il ne veut ignorer la diversité des expériences cinématographiques, des groupes et des réalisateurs qui participèrent aux débats engagés au cours des années soixante et soixante-dix.

39 En ce sens, il convient de souligner le fait que ceux qui adhérèrent à ce Mouvement étaient engagés dans le processus de transformations politiques et qu'ils faisaient de la contestation du récit de fiction commercial un instrument politique au service de la revendication d'une identité culturelle spécifique et d'un cinéma original. Ils 
privilégièrent le genre documentaire, dans toute sa diversité, depuis son expression la plus traditionnelle basée sur l'accumulation de matériel d'archive et le recueil de témoignages directs jusqu'à sa forme expérimentale à travers une réflexion sur le montage de ce matériel et sur la possibilité de l'associer à des images cinématographiques de provenance diverse, incluant même la publicité.

$\mathrm{Au}$ cours de cette première étape combative et militante, le Nouveau Cinéma Latinoaméricain s'imposa comme une arme supplémentaire dans la lutte de libération, sauvant de l'oubli la mémoire collective des peuples opprimés, dénonçant les systèmes d'oppression, reconstruisant un imaginaire social collectif, exaltant les valeurs culturelles spécifiques, légitimant l'intervention des masses, expliquant les mécanismes de domination interne et externe. Il contribua à la prise de conscience des masses. Enfin, il s'engagea à l'échelle internationale à travers le Comité du Cinéma du Tiers Monde et c'est avec l'ambition de se lier aux collectifs de cinéma progressistes et militants du monde entier qu'il organisa en 1974, au Québec, le Comité d'Action Cinématographique de Montréal qui réunit plus de 250 participants provenant de vingt-cinq pays et des cinq continents $^{22}$. L'expérience historique du Nouveau Cinéma latino-américain fut interrompue par les circonstances politiques, mais elle avait ouvert la voie à la construction d'un cinéma tiers-mondiste désireux de se renforcer dans le cadre d'une géopolitique du cinéma.

41 Traduction de l'espagnol : Jean-Paul Aubert

\section{NOTES}

1. Gilles Deleuze, La Imagen-tiempo. Estudios sobre cine 2, Piados, Barcelone, 1994.

2. In Jacques Aumont, Estética del cine, Paídos, Barcelone, 1995, pp. 82-86.

3. Il se personnifie dans l'interprétation des acteurs ou dans la bande son; il a recours notamment à l'ellipse, aux fondus, au flash-back ou au flash-forward, à la voix-off, aux effets spéciaux.

4. Pierre Sorlin, Sociología del cine. La apertura para la historia de mañana. Fondo de Cultura Económica, Mexique, 1985, pp. 50-54.

5. Fernando Birri crée en 1956 l'Institut de Cinématographie de l'Univesité Nationale du Litoral (Province de Santa Fé, Argentine). C'est dans ce cadre qu'il développera la première école documentaire en Amérique Latine. Son film Tiredié (1956-58) fonde le documentaire social.

6. La Escula Documental de Santa Fe, Documentos del Instituto de Cinematografía de la Universidad Nacional del Litoral, Rosario, Argentina, 1964, pp. 12-13.

7. Pierre Perrault (1927-1991), créateur d'un "cinéma du mot", défenseur del'identité québécoise dans la période de la "révolution tranquille". Au sein de son œuvre très abondante, produite par le National Film board, Un pays sans bon sens (1970) est peut-être le documentaire politique qui résume le mieux, à travers le recours à des voix multiples, le projet national du séparatisme québécois. 
8. Assez rapidement, le cinéma italien bénéficiera de conditions économiques meilleures du fait de son impact auprès du public, notamment dans le cadre d'une diffusion internationale, sur un marché qui dans les années d'après-guerre n'est pas encore passé sous la coupe hégémonique des monopoles américains.

9. Un soulèvement militaire avait eu lieu en juin 1973, quelques mois seulement avant le coup d'état de septembre. Les images tournées par Henrichsen et qui lui coûtèrent la vie furent révélées dans un laboratoire argentin et furent diffusées au Chili. Ces images eurent une résonance internationale.

10. L'ICAIC, Institut Cubain de l'Art et de l'Industrie Cinématographique, fondé en 1959 à Cuba.

11. Santiago Alvarez, "Motivaciones de un aniversario o respuesta inconclusa a un cuestionario que no tiene fin", in Pensamiento Crítico, n ${ }^{\circ}$, La habana, Cuba, 1970.

12. Now a obtenu le premier prix du Festival de cinéma de Leipzig en 1965.

13. Santiago Alvarez, Op. cit.

14. F. Solanas et O. Getino, Cine, cultura y descolonización, Siglo XXI, Buenos Aires, 1973, p. 88.

15. Godard, Jean Luc y Solanas, Fernando. « Godard por Solanas. Solanas por Godard », in Cine del Tercer Mundo, Nro.1, Montevideo, Uruguay, 1969. 16. http://www.lanacion.com.ar/nota.asp?nota_id=1066237

17. Paulo Antonio Paranagua, Tradición y modernidad en el cine de América Latina, Fondo de Cultura Económica, Madrid, 2003.

18. Fragment du Manifeste "L'esthétique de la faim", écrit par Glauber Rocha en 1965 et présenté lors des débats sur le Cine Novo à l'occasion de la rétrospective de Cinéma latino-américain, «Etat du Cinéma Latino-américain », organisée à Genève.

19. Le nom du groupe provient du titre de son premier long-métrage, réalisé en 1966. Ukamau signifie "c'est ainsi".

20. Jorge Sanjinés, Groupe Ukamau, Teoría y práctica de un cine junto al pueblo, Siglo XXI, México, 1980.

21. Le film fut monté à Cuba, au sein de l'ICAIC, par le réalisateur et le monteur en exil.

22. Mariano Mestman, « Entre Argel y Buenos Aires: el Comité de Cine del Tercer Mundo (1973/1974) », in Cine y fotografía como prácticas de intervención política, Université de Buenos Aires, Prometeo, 2007.

\section{RÉSUMÉS}

Cet article évalue l'apport au genre documentaire du Nouveau Cinéma Latino-américain des années soixante et soixante-dix. Dépassant l'opposition fiction-réalité, le Nouveau Cinéma Latino-américain promeut un cinéma de critique sociale grâce à des récits dont les formes varient, mais qui tous contestent la réalité de la domination impérialiste et plaident pour une réhabilitation des cultures et des identités réprimées. Ces nouveaux créateurs, engagés dans la lutte de libération de leurs peuples, sont à l'origine d' "œuvres ouvertes ", destinées à être poursuivies et achevées par l'action des nouveaux spectateurs latino-américains. 
El artículo aborda la incidencia del documental en el Nuevo Cine Latinoamericano de las décadas del ' 60 y principios de los '70. Superando la dicotomía ficción-realidad, y en una gran diversidad de discursos, el Nuevo Cine Latinoamericano impulsó un cine crítico, de sentido social, en relatos que niegan la realidad de la dominación imperialista y proponen recuperar culturas e identidades colectivas avasalladas. Estos nuevos enunciadores comprometidos con las luchas de liberación de sus pueblos, crearon "obras abiertas" que se completarían y articularían con la acción de nuevos espectadores latinoamericanos.

INDEX

Mots-clés : récit, nouveaux cinémas, critique sociale

Index chronologique : XXe siècle

\section{AUTEUR}

SUSANA SEL

Université de Buenos Aires 